\title{
Electronic and field emission properties of boron nitride/carbon nanotube superlattices
}

\author{
Vincent Meunier, ${ }^{\text {a) }}$ Christopher Roland, and J. Bernholc \\ Department of Physics, North Carolina State University, Raleigh, North Carolina 27695 \\ Marco Buongiorno Nardelli \\ Department of Physics, North Carolina State University, Raleigh, North Carolina 27695 \\ and Center for Computational Sciences (CCS) and Computational Science and Mathematics Division, \\ Oak Ridge National Laboratory, Oak Ridge, Tennessee 37830
}

(Received 19 March 2002; accepted for publication 13 May 2002)

\begin{abstract}
$\mathrm{BN} / \mathrm{C}$ nanotube superlattices are quasi one-dimensional heterostructures that show unique physical properties derived from their peculiar geometry. Using state-of-the-art ab initio calculations, we show that BN/C systems can be used for effective band-offset nanodevice engineering, polarization-based devices, and robust field emitters with an efficiency enhanced by up to two orders of magnitude over carbon nanotube systems. (c) 2002 American Institute of Physics.
\end{abstract}

[DOI: $10.1063 / 1.1491013$ ]

The discovery of carbon nanotubes as a material with outstanding mechanical and electrical properties has led to a quest for other graphene-based structures with technologically desirable properties. The closely-related boron nitride (BN) nanotubes and mixed BN/C systems, ${ }^{1-6}$ which are now being produced in gram quantities, have electronic properties that are complementary to pure carbon nanotubes and could thus be useful in a variety of electronic devices. Indeed, an early theoretical study suggested BN/C junctions as a potentially practical way to realize stable, nanoscale heterojunctions. ${ }^{7}$ Very recently, BN nanotubes have been predicted to possess nonzero spontaneous polarization fields, ${ }^{8,9}$ analogous to that in the well-known wurtzite semiconductors. This letter investigates BN/C superlattices and heterojunctions through large-scale $a b$ initio simulations. ${ }^{10}$ The results provide accurate values of valence and conduction band offsets and polarization fields. In particular, we show by explicit calculations that the spontaneous polarization fields present in BN/C heterostructures could dramatically enhance field emission properties and lead to electronic devices.

Since BN/C nanotube superlattices are actually quasi one-dimensional (1D) heterojunctions, their electronic properties are first and foremost characterized by their band offsets. ${ }^{11}$ Pure BN nanotubes are wide band gap semiconductors with a band gap of $5.5 \mathrm{eV}$. Carbon nanotubes, in turn, may be either metallic or semiconducting, depending on their indices $(\ell, m)$. The $(\ell, 0)$ zigzag tubes (on which we will concentrate here) with $\ell=3 n$ ( $n$ integer) will be metallic. For BN/C nanotube systems, the valence and conduction band offsets are spatially direct, with typical values given in Table I. From this table, it is clear that simple matching of two straight nanotubes will give rise to a variety of band alignments with band offsets that are sensitive to the helicity and radii of the tubes. In particular, for metallic carbon nano-

\footnotetext{
a) Author to whom correspondence should be addressed; electronic mail: meunierv@ornl.gov
}

tubes, the heterojunction will behave as a Schottky diode. Thus, combining the $\mathrm{BN}$ and carbon nanotubes of various helicities offers a broad range of opportunities for bandoffset engineering and for the construction of different nanotubular heterojunctions.

Turning to the more unique aspects of the electronic properties, the majority of $\mathrm{BN} / \mathrm{C}$ nanotube superlattices are characterized by a spontaneous polarization field, which is a direct consequence of the polar nature of the $\mathrm{B}-\mathrm{N}$ bond and the low symmetry of the underlying lattice. In a periodic superlattice of polar and nonpolar materials (such as $\mathrm{BN}$ and C), any spontaneous polarization field will manifest itself in the behavior of the total electrostatic potential of the system. In fact, the latter displays a typical sawtooth behavior that is the signature of the presence of a spontaneous polarization field superimposed onto the periodic crystalline potential. ${ }^{14}$ For convenience, we have computed the planar average of the electrostatic potential along the nanotube axis, see Fig. 1(a). This average, which is over the directions perpendicular to the tube axis, displays strong oscillations due to the varying strength of the ionic potentials. To subtract out this effect, we have implemented the procedure of Ref. 12, and thereby calculated the one-dimensional macroscopic average of the electrostatic potential of the system. The value of the polarization field is then obtained from the slope of the macroscopic average potential ${ }^{13,14}$ and is shown in Fig. 1(b).

It is clear that the symmetry of the nanotube plays an important role in determining the magnitude of the spontaneous polarization field. The strongest effects will be observed in the $(\ell, 0)$ zigzag nanotubes, since this geometry maximizes the dipole moment of the $\mathrm{B}-\mathrm{N}$ bond. Hence, we

TABLE I. Conduction $\left(\Delta E_{c}\right)$ and valence $\left(\Delta E_{v}\right)$ band offsets for a set of zigzag BN/C heterostructures, calculated using the vacuum level as reference (see Ref. 11).

\begin{tabular}{lcccc}
\hline \hline$(\mathrm{eV})$ & $(7,0)$ & $(8,0)$ & $(9,0)$ & $(10,0)$ \\
\hline$\Delta E_{c}$ & -2.09 & -2.50 & -2.42 & -2.52 \\
$\Delta E_{v}$ & 0.82 & 1.12 & 1.53 & 1.46 \\
\hline \hline
\end{tabular}



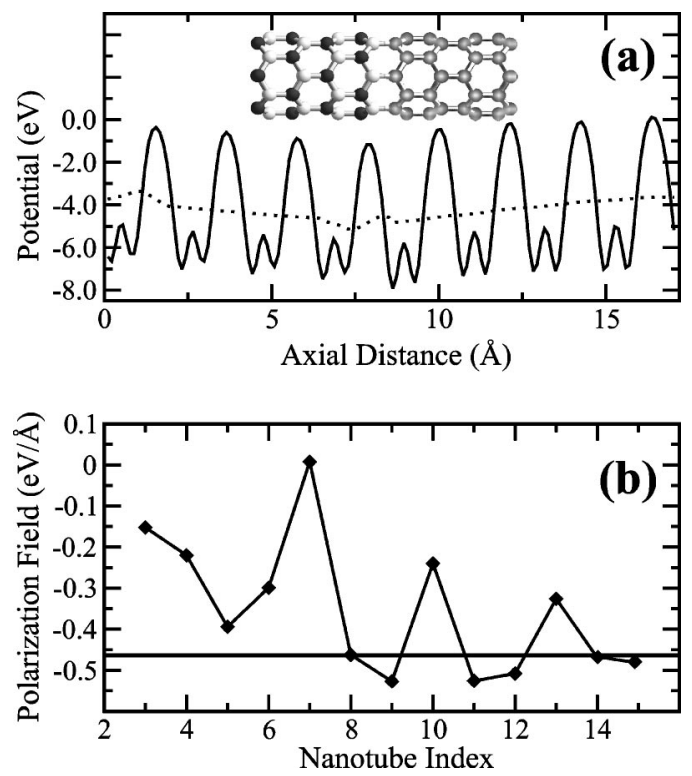

FIG. 1. (a) Average electrostatic potential (full) and macroscopic average (dotted) along a BN/C $(6,0)$ superlattice (insert) and (b) macroscopic polarization field for zigzag $(\ell, 0)$ tubes as a function of the $\ell$ index (the straight segments are drawn as guide for the eye). The asymptotic value for planar $\mathrm{BN}$ is represented by the horizontal line.

have primarily concentrated on zigzag nanotubes with diameters of up to $11.74 \AA$, and the flat sheet as the limit for large diameter tubes. The corresponding spontaneous polarization fields are summarized in Fig. 1(b). In contrast, the $(\ell, \ell)$ armchair nanotubes are not expected to display any spontaneous polarization field. This is because any individual nanotube ring must be charge neutral, so that no fields are possible. We have directly verified this for the case of a $(5,5) \mathrm{BN} / \mathrm{C}$ superlattice. Chiral nanotubes will have fields that are between the zigzag and armchair values.

From Fig. 1(b), it is clear that the magnitude of the macroscopic electric field for the various superlattices has a strong oscillatory character. Nanotubes with indices 7, $10, \ldots 3 n+1$ are characterized by a substantially smaller polarization field. This effect originates from the fact that the carbon sections-located between the $\mathrm{BN}$ ones-will screen the macroscopic polarization field with an efficiency that depends on the nanotube helicity. From the band offsets, it follows that the valence state of the BN/C superlattices is always spatially localized in the carbon region. This state plays an important role in determining the response to the macroscopic electric field induced by the $\mathrm{BN}$ section. In particular, the oscillatory behavior observed as a function of the helicity index can be understood from the symmetry property of the valence state, displayed in Fig. 2 for two zigzag nanotubes. Figure 2 directly shows that the valence state is localized within the carbon section. For small diameter nanotubes it displays either a longitudinal or a transverse symmetry as exemplified by the $(7,0)$ and $(8,0)$ tubes, respectively. ${ }^{15}$ Specifically, for $(\ell=3 n+1,0)$ nanotubes, such as the $(7,0)$ tube, the valence state always assumes a longitudinal character. The axial distribution of valence electrons will therefore induce a depolarization field that is opposite to the one intrinsic to the BN section. This field is quite effective in small diameter nanotubes in reducing the polarization, so that for the $(7,0)$ nanotube the screening is almost total and the ob-

Downloaded 28 Feb 2008 to 152.1.211.43. Redistribution subject
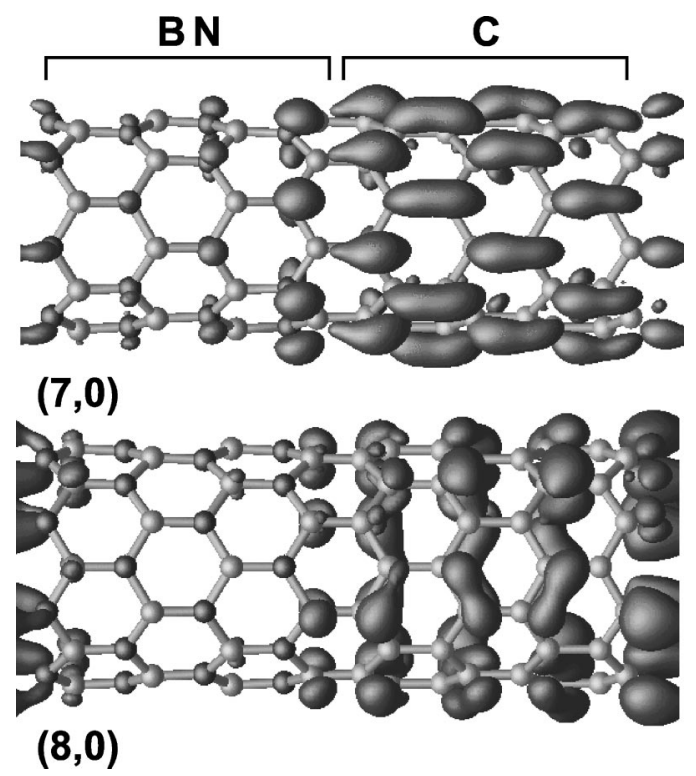

FIG. 2. Three-dimensional plots of the valence state for $(7,0)$ and $(8,0)$ $\mathrm{BN} / \mathrm{C}$ superlattices.

served macroscopic field is close to zero. For larger diameter nanotubes, the symmetry of the valence state gradually loses its peculiar axial or longitudinal character and the macroscopic field asymptotically approaches the value obtained for a flat BN sheet in the "zigzag" direction.

Despite the screening by the valence electrons distributed over the carbon portion of the BN/C superlattices, a net polarization field is built up along any zigzag structure. The existence of an intrinsic macroscopic field will clearly influence the extraction of electrons from BN/C systems, when these are used as field emitting devices. Qualitatively, a good electron emitter is characterized by a large geometrical field enhancement factor $\beta$ and a small work function $\phi$ that is an intrinsic property of the emitter material. Quantitatively, the current at the emitter tip is calculated from the FowlerNordheim relationship. ${ }^{16}$

To assess the efficacy of BN/C systems as emitters, we have computed the field enhancement factor for tubes of length $15.62 \AA$ in an applied field of $0.11 \mathrm{~V} / \AA$. As expected, the applied field is very well screened inside the system and a local field enhancement factor of $\sim 2.1$ is obtained for the different BN/C systems. Since the field enhancement factor increases linearly with the size of the nanotubes, large enhancement factors are clearly possible for long nanotubes. ${ }^{17,18}$

Although carbon nanotubes are already considered to be good emitters, these desirable properties may be further enhanced by making use of the electronic properties of $\mathrm{BN} / \mathrm{C}$ systems. The idea here is to make use of polarization fields in order to reduce the work function of the tips, thereby enhancing the extraction of electrons from the system.

In order to examine this effect quantitatively, we have built up a set of finite sized $(6,0)$ zigzag structures using B, $\mathrm{N}$, and $\mathrm{C}$ in various combinations. The work functions were then computed as the difference between the vacuum level and the Fermi energy of the system. The former was obtained by the previously discussed potential average procedure, while the latter was simply given by the highest occupied AIP license or copyright; see http://apl.aip.org/apl/copyright.jsp 
eigenstate of the system. In order to avoid any spurious effects arising from the periodic calculation, we have determined that the unit cell should have a vacuum of at least 15 $\AA$ on both sides of the system along its axis. ${ }^{19}$ With this method, we calculated a work function of $5.01 \mathrm{eV}$ for a $(10,10)$ carbon nanotube, in close agreement with previously published values. ${ }^{20,21}$ For the smaller diameter $(6,0)$ carbon nanotube, we found a considerably larger work function of $6.44 \mathrm{eV}$. This is primarily due to the mixing of the $\sigma$ and $\pi$ valence bands in this highly curved tube.

Turning to the heterostructures, we consider a finite $\mathrm{NB} / \mathrm{C}(6,0)$ system consisting of four alternating $\mathrm{N}$ and $\mathrm{B}$ layers followed by $8 \mathrm{C}$ layers, giving a total of 96 atoms. Due to the net polarization field experienced by the electron, the work function is reduced to $5.04 \mathrm{eV}$ at the $\mathrm{C}$ tip and increased to $7.52 \mathrm{eV}$ at the $\mathrm{N}$ tip. The same trend is observed for the BN/C system, for which the work function at the B tip is equal to $5.00 \mathrm{eV}$ while it takes a value of $6.45 \mathrm{eV}$ at the $\mathrm{C}$ tip. The work function is therefore decreased by a significant $1.40 \mathrm{eV}$, as compared to the pure carbon system. This is large enough to lead to significant macroscopic effects. According to the Fowler-Nordheim relationship, the logarithm of the current density $(J)$ depends upon the work function $\phi$ as $\ln J \sim-\phi^{3 / 2}$. It follows that the insertion of BN segments in $\mathrm{C}$ nanotubes will increase the current density by up to two orders of magnitude as compared to pure carbon nanotube systems.

In summary, we have shown that a wide variety of nanoscale heterojunctions may be formed with BN/C structures, with the possibility of band-offset engineering. BN/C junctions and superlattices are characterized by the presence of a spontaneous polarization field, whose value is highly sensitive to the helicity of the underlying nanotubes. The polarization field can be used to lower the work function of BN/C nanotube tips, thereby increasing the field emitting properties over carbon nanotube tips by up to two orders of magnitude.

The authors would like to thank R. Resta and S. Nakhmanson for scientific discussions. This work was supported in part by NASA, DOE, and ONR. We also thank the DOD and NC Supercomputing Centers for extensive computer support.

${ }^{1}$ A. Rubio, J. L. Corkill, and M. L. Cohen, Phys. Rev. B 49, 5081 (1994).

${ }^{2}$ N. G. Chopra, R. J. Luyken, K. Cherrey, V. H. Crespi, M. L. Cohen, S. G. Louie, and A. Zettl, Science 269, 966 (1995).

${ }^{3}$ O. Stephan, P. M. Ajayan, C. Colliex, P. Redlich, J. M. Lambert, P. Bernier, and P. Lefin, Science 266, 1683 (1994).

${ }^{4}$ A. Loiseau, F. Willaime, N. Demoncy, G. Hug, and H. Pascard, Phys. Rev. Lett. 76, 4737 (1996).

${ }^{5}$ J. Cumings and A. Zettl, Chem. Phys. Lett. 316, 211 (2000).

${ }^{6}$ R. S. Lee, J. Gavillet, M. L. de la Chapelle, A. Loiseau, J.-L. Cochon, D. Pigache, and J. T. F. Willaime, Phys. Rev. B 64, 121405 (2001).

${ }^{7}$ See for example: X. Blase, J. C. Charlier, A. DeVita, and R. Car, Appl. Phys. Lett. 70, 197 (1997).

${ }^{8}$ E. J. Mele, P. Král, and D. Tomanek, http://www.aps.org/meet/MAR01/ baps/abs/S8658005.html; Phys. Rev. Lett. 88, 056803 (2002).

${ }^{9}$ M. Buongiorno Nardelli and J. Bernholc http://www.aps.org/meet/ MAR01/baps/abs/S3500012.html; S. Nakhmanson, V. Meunier, J. Bernholc, and M. Buongiorno Nardelli (unpublished).

${ }^{10}$ The $a b$ initio calculations were carried out via a combination of standard multigrid-based real-space [E. L. Briggs, D. J. Sullivan, and J. Bernhole, Phys. Rev. B 52, R5471 (1995); 54, 14362 (1996)] and plane-wave methods [S. Baroni et al., http://www.pwscf.org]. After a careful minimization of the atomic coordinates, the structural parameters and electronic band structures obtained were in perfect agreement with those of previous calculations (see Ref. 7).

${ }^{11}$ In contrast to three-dimensional solids, the asymptotic band offsets of such heterojunctions are conveniently computed from the bulk properties of their isolated components, since the vacuum level (i.e., the ionization potential) may be used as an unequivocal reference energy [R. Resta (private communication); see also L. Kleinman, Phys. Rev. B 24, 7412 (1981)].

${ }^{12}$ A. Baldereschi, S. Baroni, and R. Resta, Phys. Rev. Lett. 61, 734 (1988).

${ }^{13}$ In 1D systems, the volume of the cell is not relevant to the evaluation of averages and for that reason we have defined the effective volume as being the one in which $99 \%$ of the total charge is located (see Ref. 9).

${ }^{14}$ M. Posternak, A. Baldereschi, A. Catellani, and R. Resta, Phys. Rev. Lett. 64, 1777 (1990)

${ }^{15}$ C. L. Kane and E. J. Mele, Phys. Rev. B 59, R12759 (1999).

${ }^{16}$ R. H. Fowler and L. W. Nordheim, Proc. R. Soc. London, Ser. A 119, 173 (1928).

${ }^{17}$ S. Han and J. Ihm, Phys. Rev. B 61, 9986 (2000).

${ }^{18}$ A. Maiti, C. Brabec, C. Roland, and J. Bernholc, Phys. Rev. Lett. 73, 2468 (1994); C. Brabec, A. Maiti, C. Roland, and J. Bernholc, Phys. Rev. B 52, 14850 (1995).

${ }^{19}$ We used the so-called dipole correction in order to avoid nondesirable supercell effects and to ensure the flatness of the electrostatic potential in the vacuum [L. Bengtsson, Phys. Rev. B 59, 12301 (1999)].

${ }^{20}$ M. Shiraishi and M. Ata, Carbon 39, 1913 (2001).

${ }^{21}$ G. Zhou, W. H. Duan, and B. L. Gu, Phys. Rev. Lett. 87, 095504 (2001). 